ADP-01-56/T488

\title{
Quenched chiral perturbation theory for baryon form factors
}

\author{
Derek B. Leinweber ${ }^{\text {a }}$

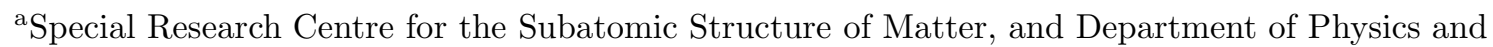 \\ Mathematical Physics, University of Adelaide, SA, 5005, Australia
}

\begin{abstract}
A new intuitive method for the rapid calculation of the leading nonanalytic behavior of hadronic observables in quenched chiral perturbation theory is presented. After proving the technique in a consideration of baryon masses, the quenched magnetic moments of octet baryons are addressed. The technique provides a separation of magnetic moment contributions into full, sea, valence and quenched valence contributions, the latter being the conventional view of the quenched approximation. Both baryon mass and meson mass violations of SU(3)-flavor symmetry are accounted for. A comprehensive examination of the individual quark-sector contributions to octet baryon magnetic moments reveals that the $u$-quark contribution to the proton magnetic moment provides an optimal opportunity to directly view nonanalytic behavior associated with the quenched meson cloud of baryons in the quenched approximation.
\end{abstract}

\section{INTRODUCTION}

Separation of the valence and sea-quark-loop contributions to the meson cloud of full QCD hadrons is a non-trivial task. Early calculations addressing the meson cloud of mesons employed a diagrammatic method [1]. The formal theory of quenched chiral perturbation theory $(\mathrm{Q} \chi \mathrm{PT})$ was subsequently established in Ref. [2]. There, meson properties were examined in a formulation where extra commuting ghost-quark fields are introduced to eliminate the dependence of the path integral on the fermion-matrix determinant. This approach was extended to the baryon sector in Ref. [3].

While the formalism of $\mathrm{Q} \chi \mathrm{PT}$ is essential to establishing the field theoretic properties, it is desirable to formulate an efficient and perhaps more intuitive approach to the calculation of quenched chiral coefficients. Rather than introduce extra degrees of freedom to remove the effects of seaquark loops, the approach described herein describes the systematic separation of valence and sea-quark-loop contributions. Upon removing the contributions of sea-quark-loops, one arrives at the conventional view of quenched chiral perturbation theory.

Since the presentation of this talk, there has been a resurgence in $\mathrm{Q} \chi \mathrm{PT}$ calculations. In particular, the magnetic moments of octet baryons have been examined [ [4] using the formal approach of $\mathrm{Q} \chi \mathrm{PT}$ [3]. There the leading-nonanalytic (LNA) behavior of the magnetic moment for each baryon of the octet is calculated. The formal approach completely eliminates all sea-quark-loop contributions to baryon moments.

However, sea-quark loops do make a contribution to matrix elements in the quenched approximation. Insertion of the current in calculating the three-point correlation function provides pair(s) of quark-creation and annihilation operators. These can be contracted with the quark field operators of the hadron interpolating fields providing "connected insertions" of the current, or self-contracted to form a sea-quark-loop contribution or "disconnected insertion" of the current. The latter contributions to baryon electromagnetic form factors have already been examined in quenched simulations [5, 6]. Hence in formulating quenched chiral perturbation theory it is important to provide an opportunity to include these particular sea-quark-loop contributions.

In the following, a new intuitive method is presented for the rapid calculation of the quenched chiral coefficients of the LNA terms of QCD. While the quenched $\eta^{\prime}$ also gives rise to new non- 
analytic behavior, such effects on baryon magnetic moments are numerically small in the region of interest [4] and are not addressed further in this brief report. Sec. 2 proves the technique via a consideration of baryon masses. The derivation of the quenched chiral coefficients for the quenched magnetic moments of octet baryons is described in Sec. 3. The technique provides a separation of magnetic moment contributions into full, sea, valence and quenched valence contributions, the latter being the conventional view of the quenched approximation. Sec. 3 also outlines how both baryon mass and meson mass violations of SU(3)-flavor symmetry are accounted for. A comprehensive examination of the individual quark-sector contributions to octet baryon magnetic moments is presented in Sec. 4 .

\section{QUENCHED BARYON MASSES}

The following calculations are simplified through the use of the standard octet and singlet interaction Lagrangians [7] in which mesonbaryon couplings are subsequently expressed in terms of the $F$ and $D$ coupling coefficients. For example, $f_{N N \pi}=F+D, f_{\Sigma N K}=D-F$, etc. In the following, numerical estimates are based on the one-loop corrected values [8] of $F=0.40$ and $D=0.61$, with $f_{\pi}=f_{K}=93 \mathrm{MeV}$.

The quark flow diagrams of Fig. 1 illustrate the processes which give rise to the LNA behavior of proton observables. Table 1 summarizes the contributions of the $\pi-, \eta$ - and $\eta^{\prime}$-cloud diagrams of Fig. 1 labeled by the corresponding quark-flow diagrams. The suppression of sea-quark-loops in the quenched approximation renders the $\eta^{\prime}$ mass degenerate with the pion. The octet-decuplet transition couplings are taken to be negligible. Summation of these couplings and incorporation of the factors from the loop integral provides the LNA term

$-\left(3 F^{2}+D^{2}\right) \frac{m_{\pi}^{3}}{8 \pi f_{\pi}^{2}}=-3.92 m_{\pi}^{3}$.

The separation of the meson cloud into valence and sea-quark contributions is shown in the quark-flow diagrams labeled by letters. To quench the theory, one must understand the
Table 1

Meson-cloud contributions of Fig. 1.

\begin{tabular}{cccc}
\hline Fig. & Channel & Coupling & Coupling \\
\hline \hline a,b,c & $p \pi^{0}$ & $f_{N N \pi}^{2}$ & $(F+D)^{2}$ \\
a,b,c & $p \eta$ & $f_{N N \eta}^{2}$ & $(3 F-D)^{2} / 3$ \\
a,b,c & $p \eta^{\prime}$ & $f_{N N \eta^{\prime}}^{2}$ & $2(3 F-D)^{2} / 3$ \\
f,g & $n \pi^{+}$ & $2 f_{N N \pi}^{2}$ & $2(F+D)^{2}$ \\
\hline
\end{tabular}

Table 2

Sea-quark-loop contributions of Fig. 1.

\begin{tabular}{cccc}
\hline Fig. & Channel & Coupling & Coupling \\
\hline \hline $\mathrm{b}$ & $\Lambda K^{+}$ & $f_{\Lambda N K}^{2}$ & $(3 F+D)^{2} / 3$ \\
$\mathrm{~b}$ & $\Sigma^{0} K^{+}$ & $f_{\Sigma N K}^{2}$ & $(D-F)^{2}$ \\
$\mathrm{c}$ & $\Sigma^{+} K^{0}$ & $2 f_{\Sigma N K}^{2}$ & $2(D-F)^{2}$ \\
$\mathrm{e}$ & $\Sigma^{+} K^{0}$ & $2 f_{\Sigma N K}^{2}$ & $2(D-F)^{2}$ \\
$\mathrm{~g}$ & $\Lambda K^{+}$ & $f_{\Lambda N K}^{2}$ & $(3 F+D)^{2} / 3$ \\
$\mathrm{~g}$ & $\Sigma^{0} K^{+}$ & $f_{\Sigma N K}^{2}$ & $(D-F)^{2}$ \\
\hline
\end{tabular}

chiral behavior of the valence-quark loops of Figs. 1 (a), (d) and (f) and the sea-quark loops of Figs. 1 (b), (c), (e), (g), (h) and (i) separately. If one can isolate the behavior of the diagrams involving a quark loop, then one can use the known LNA behavior of the full meson-based diagrams to extract the corresponding valence-loop contributions.

For example, Fig. 1(b) involves a $u$-quark loop where no exchange term is possible. Thus the $u$ quark in the loop is distinguishable from all the other quarks in the diagram. The chiral structure of this diagram is therefore identical to that for a "strange" quark loop, as illustrated in Fig. 1 $(\mathrm{h})$, provided the "strange" quark in this case is understood to have the same mass as the $u$-quark.

The corresponding hadron diagram which gives rise to the LNA structure of Fig. I $1(\mathrm{~b})$ is therefore the $K^{+}$-loop diagram above Fig. 1 (h), with the distinguishable "strange" quark mass set equal to the mass of the $u$-quark. That is, the intermediate baryon masses appearing in the $\mathrm{K}^{+}$-loop diagram are degenerate with the nucleon. Similarly, the "kaon" mass is degenerate with the pion.

The sum of the first two lines of Table 2 pro- 

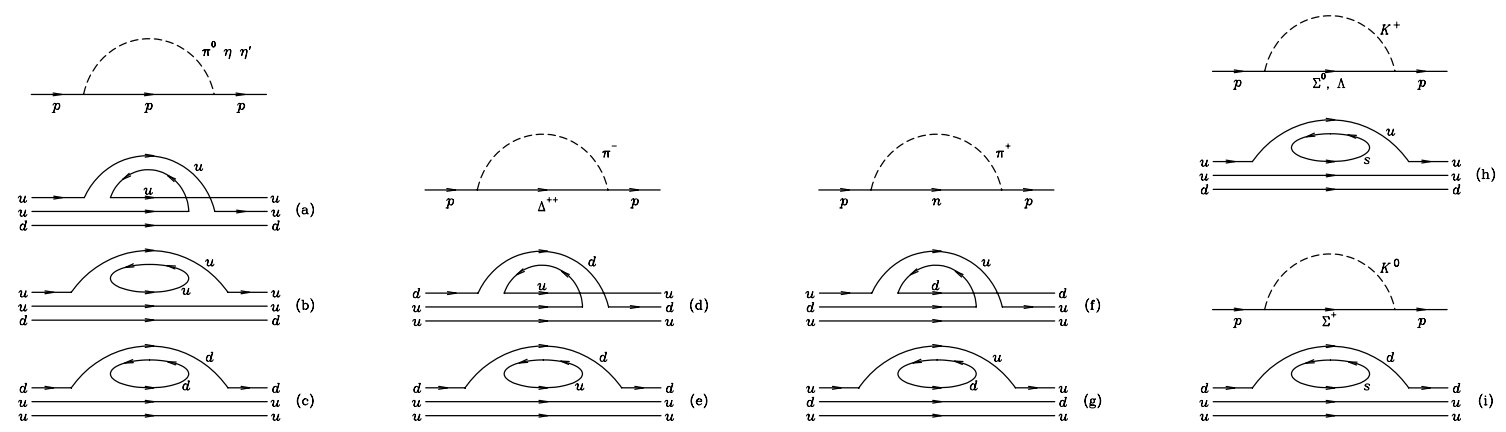

Figure 1. The pseudo-Goldstone meson cloud of the proton and the associated quark flow diagrams.

vides the contribution of diagram Fig. 11(b). Similar arguments allow one to establish the remaining loop contributions to the light-meson cloud. Summing the couplings of Table 2 indicates seaquark-loops contribute

$$
-\left(9 F^{2}-6 F D+5 D^{2}\right) \frac{m_{\pi}^{3}}{24 \pi f_{\pi}^{2}}=-2.82 m_{\pi}^{3},
$$

to the LNA behavior of the nucleon such that the net quenched contribution is

$-\left(3 F D-D^{2}\right) \frac{m_{\pi}^{3}}{12 \pi f_{\pi}^{2}}=-1.10 m_{\pi}^{3}$,

in agreement with the more formal approach of Labrenz and Sharpe [3].

\section{BARYON MAGNETIC MOMENTS}

The LNA contribution to baryon magnetic moments proportional to $m_{\pi}$ or $m_{K}$ has its origin in couplings of the electromagnetic (EM) current to the meson propagating in the intermediate meson-baryon state. In order to pick out a particular quark-flavour contribution, one sets the electric charge for the quark of interest to one and the charge of all other flavours to zero.

Table 3 reports results for the $u$-quark in the proton. The total contributions are calculated in the standard way, but with charge assignments for the intermediate mesons (indicated in the Charge column) reflecting in this case $q_{u}=1$ and $q_{d}=q_{s}=0$. The extra baryon subscripts on the meson masses are a reminder of the baryons participating in the diagram to facilitate more sophisticated treatments of the loop integral in which baryon mass splittings are taken into account. The LNA contribution is $\beta \frac{m_{N}}{8 \pi f_{\pi}^{2}} m_{\pi} \equiv$ $\chi m_{\pi}$ with $\beta$ and $\chi$ indicated in the last two columns.

"Sea-quark-loop contributions" are contributions in which the EM current couples to a seaquark loop, in this case a $u$ quark. Using the techniques described in Sec. 2, one can calculate the contributions of these loops alone to the baryon magnetic moment. The Mass column of Table 3 is a reminder that the mass of the "kaon" considered in determining the coupling is actually the pion mass for diagrams 1(b) and (e). These diagrams will contribute, even in the quenched approximation, when disconnected insertions of the EM current are included in simulations [5,6].

Subtracting the sea-quark-loop contributions from the total contributions provides the valence contribution of full QCD. "Quenching considerations" focus on diagrams in which the EM current couples to a valence quark in a meson composed with a sea-quark loop. Subtracting off these couplings from the valence contribution provides the quenched valence contribution.

\section{RESULTS}

This approach allows one to separate an individual quark-flavour contribution into four categories, namely: full, loop, valence, and quenched valence contributions. The LNA loop contribution is relevant to disconnected insertions of the EM current in either full or quenched QCD, 
Table 3

Determination of the $u$-quark contribution to the proton magnetic moment as illustrated in Fig. 1

Total Contributions

\begin{tabular}{ccccccc}
\hline Diagram & Channel & Mass & Charge & Term & $\beta$ & $\chi$ \\
\hline \hline $\mathrm{f}, \mathrm{g}$ & $n \pi^{+}$ & $N \pi$ & +1 & $+2 f_{N N \pi}^{2} m_{\pi}$ & $-(F+D)^{2}$ & -4.41 \\
$\mathrm{~h}$ & $\Sigma^{0} K^{+}$ & $\Sigma K$ & +1 & $+f_{\Sigma N K}^{2} m_{N \Sigma K}$ & $-(D-F)^{2} / 2$ & -0.10 \\
$\mathrm{~h}$ & $\Lambda K^{+}$ & $\Lambda K$ & +1 & $+f_{\Lambda N K}^{2} m_{N \Lambda K}$ & $-(3 F+D)^{2} / 6$ & -2.36 \\
\hline
\end{tabular}

Sea-Quark Loop Contributions

\begin{tabular}{cccclcc}
\hline Diagram & Channel & Mass & Charge & Term & $\beta$ & $\chi$ \\
\hline \hline $\mathrm{b}$ & $\Lambda K^{+}$ & $N \pi$ & -1 & $-f_{\Lambda N K}^{2} m_{\pi}$ & $(3 F+D)^{2} / 6$ & +2.36 \\
$\mathrm{~b}$ & $\Sigma^{0} K^{+}$ & $N \pi$ & -1 & $-f_{\Sigma N K}^{2} m_{\pi}$ & $(D-F)^{2} / 2$ & +0.10 \\
$\mathrm{e}$ & $\Sigma^{+} K^{0}$ & $N \pi$ & -1 & $-2 f_{\Sigma N K}^{2} m_{\pi}$ & $(D-F)^{2}$ & +0.19 \\
Total & & & & & & +2.65 \\
\hline
\end{tabular}

Net Valence Contribution: $\quad-7.06 m_{\pi}-0.10 m_{N \Sigma K}-2.36 m_{N \Lambda K}$

Quenching Considerations

\begin{tabular}{cccclcc}
\hline Diagram & Channel & Mass & Charge & Term & $\beta$ & $\chi$ \\
\hline \hline $\mathrm{b}$ & $\Lambda K^{+}$ & $N \pi$ & +1 & $+f_{\Lambda N K}^{2} m_{\pi}$ & $-(3 F+D)^{2} / 6$ & -2.36 \\
$\mathrm{~b}$ & $\Sigma^{0} K^{+}$ & $N \pi$ & +1 & $+f_{\Sigma N K}^{2} m_{\pi}$ & $-(D-F)^{2} / 2$ & -0.10 \\
$\mathrm{~g}$ & $\Lambda K^{+}$ & $N \pi$ & +1 & $+f_{\Lambda N K}^{2} m_{\pi}$ & $-(3 F+D)^{2} / 6$ & -2.36 \\
$\mathrm{~g}$ & $\Sigma^{0} K^{+}$ & $N \pi$ & +1 & $+f_{\Sigma N K}^{2} m_{\pi}$ & $-(D-F)^{2} / 2$ & -0.10 \\
$\mathrm{~h}$ & $\Sigma^{0} K^{+}$ & $\Sigma K$ & +1 & $+f_{\Sigma N K}^{2} m_{N \Sigma K}$ & $-(D-F)^{2} / 2$ & -0.10 \\
$\mathrm{~h}$ & $\Lambda K^{+}$ & $\Lambda K$ & +1 & $+f_{\Lambda N K}^{2} m_{N \Lambda K}$ & $-(3 F+D)^{2} / 6$ & -2.36 \\
\hline
\end{tabular}

Net Quenched Valence Contribution: $-2.15 m_{\pi}$

whereas the LNA valence contribution is relevant to connected insertions of the EM current only in full QCD. The final category of quenched valence contributions is relevant to connected insertions of the EM current in quenched QCD. The latter is often referred to as the quenched QCD result.

Similar arguments allow one to calculate the contributions of all quark flavors. Table 1 reports values for the coefficient, $\chi$, providing the LNA contribution to baryon magnetic moments $\left(\chi m_{\pi}\right.$ or $\chi m_{K}$ as appropriate) by quark sectors normalized to unit charge. Charge symmetry provides the contributions for the other baryons and $\Sigma^{0}$ is the isospin average of $\Sigma^{+}$and $\Sigma^{-}$. Quenched coefficients for $\Lambda$ are determined via $\mathrm{SU}(3)$-flavour symmetry.

Baryon moments are constructed from the quark sector coefficients by multiplying the $u, d$ and $s$ results by their appropriate charge factors and summing. For example, the proton moment is $\mu_{p}=2 u_{p} / 3-d_{p} / 3-s_{p} / 3$ and the neutron moment is $\mu_{n}=-u_{p} / 3+2 d_{p} / 3-s_{p} / 3$.

These results reveal that the $u$-quark in the proton, known to have small statistical errors [10], provides an excellent opportunity to directly view nonanalytic behavior associated with the quenched meson cloud of baryons in the quenched approximation. Figure 2 illustrates the anticipated curvature [9] based on the quenched valence results presented here.

\section{ACKNOWLEDGEMENTS}

Thanks to Matthias Burkardt, Tony Thomas, Tony Williams and Ross Young for beneficial discussions. This research is supported by the Australian Research Council. 


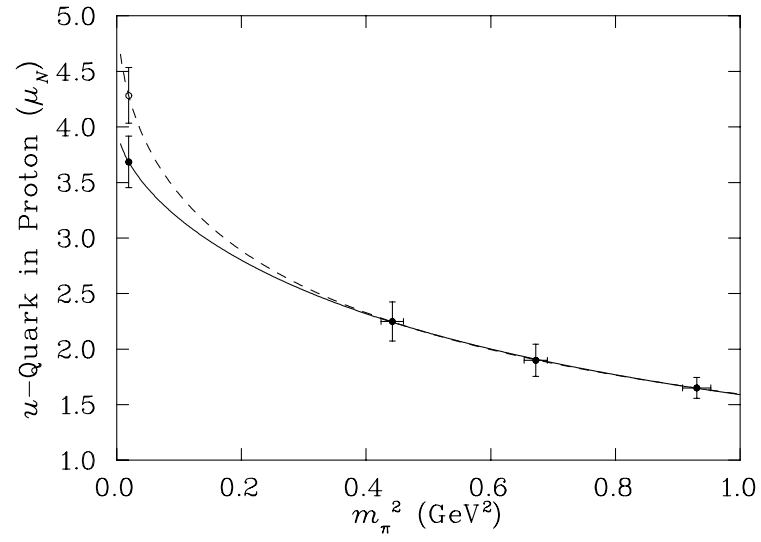

Figure 2. Chiral extrapolation via the Padé of [9]. The solid curve displays the self-consistent extrapolation of the quenched simulation results of [10], whereas the dashed curve shows the curvature of full QCD.

\section{REFERENCES}

1. S. R. Sharpe, Phys. Rev. D 46 (1992) 3146 hep-lat/9205020, ibid. 41 (1990) 3233.

2. C. W. Bernard and M. F. Golterman, Phys. Rev. D 46 (1992) 853 hep-lat/9204007.

3. J. N. Labrenz and S. R. Sharpe, Phys. Rev. D 54 (1996) 4595 hep-lat/9605034.

4. M. J. Savage, nucl-th/0107038.

5. S. J. Dong, K. F. Liu and A. G. Williams, Phys. Rev. D 58 (1998) 074504 hep$\mathrm{ph} / 9712483$.

6. W. Wilcox, Nucl. Phys. Proc. Suppl. 94 (2001) 319 hep-lat/0010060.

7. T. A. Rijken, V. G. Stoks and Y. Yamamoto, Phys. Rev. C 59 (1999) 21 nucl-th/9807082.

8. E. Jenkins, et al., Phys. Lett. B 302 (1993) 482 [Erratum-ibid. B 388 (1993) 866] hep$\mathrm{ph} / 9212226$.

9. D. B. Leinweber, D. H. Lu and A. W. Thomas, Phys. Rev. D 60 (1999) 034014 hep-lat/9810005; E. J. HackettJones, D. B. Leinweber and A. W. Thomas, Phys. Lett. B $\mathbf{4 8 9}$ (2000) 143 hep lat/0004006].

10. D. B. Leinweber, R. M. Woloshyn and T. Draper, Phys. Rev. D 43 (1991) 1659.
Table 4

Coefficients, $\chi$, providing the LNA contribution to baryon magnetic moments by quark sectors normalized to unit charge. Intermediate (Int.) meson-baryon channels are indicated to allow for $\mathrm{SU}(3)$-flavour breaking in both the meson and baryon masses. Total, sea-quark loop, valence (Val.) and quenched valence (Qch.) coefficients are indicated. Note $\epsilon=0.0004$.

\begin{tabular}{|c|c|c|c|c|c|}
\hline$q$ & Int. & Total & Loop & Val. & Qch. \\
\hline \multirow[t]{3}{*}{$u_{p}$} & $N \pi$ & -4.41 & +2.65 & -7.06 & -2.15 \\
\hline & $\Lambda K$ & -2.36 & 0 & -2.36 & 0 \\
\hline & $\Sigma K$ & -0.10 & 0 & -0.10 & 0 \\
\hline \multirow{2}{*}{$d_{p}$} & $N \pi$ & +4.41 & +2.65 & +1.76 & +2.15 \\
\hline & $\Sigma K$ & -0.19 & 0 & -0.19 & 0 \\
\hline \multirow[t]{2}{*}{$s_{p}$} & $\Lambda K$ & +2.36 & +2.36 & 0 & 0 \\
\hline & $\Sigma K$ & +0.29 & +0.29 & 0 & 0 \\
\hline \multirow[t]{4}{*}{$u_{\Sigma^{+}}$} & $\Sigma \pi$ & -1.38 & +1.38 & -2.77 & 0 \\
\hline & $\Lambda \pi$ & -1.07 & +1.07 & -2.15 & 0 \\
\hline & $N K$ & 0 & +0.19 & -0.19 & -0.19 \\
\hline & $\Xi K$ & -4.41 & 0 & -4.41 & -1.95 \\
\hline \multirow{3}{*}{$d_{\Sigma^{+}}$} & $\Sigma \pi$ & +1.38 & +1.38 & 0 & 0 \\
\hline & $\Lambda \pi$ & +1.07 & +1.07 & 0 & 0 \\
\hline & $N K$ & +0.19 & +0.19 & 0 & 0 \\
\hline \multirow[t]{2}{*}{$s_{\Sigma^{+}}$} & $N K$ & -0.19 & 0 & -0.19 & +0.19 \\
\hline & $\Xi K$ & +4.41 & +2.46 & +1.95 & +1.95 \\
\hline \multirow[t]{4}{*}{$u_{\Lambda} \mid d_{\Lambda}$} & $\Sigma \pi$ & +0.00 & +1.07 & -1.07 & +0.00 \\
\hline & $\Lambda \eta_{l}$ & +0.00 & $\epsilon$ & $-\epsilon$ & +0.00 \\
\hline & $N K$ & +2.36 & +1.57 & +0.79 & +0.79 \\
\hline & $\Xi K$ & -0.25 & +0.00 & -0.25 & +0.29 \\
\hline \multirow[t]{3}{*}{$s_{\Lambda}$} & $\Lambda \eta_{s}$ & +0.00 & +1.57 & -1.57 & +0.00 \\
\hline & $N K$ & -4.72 & +0.00 & -4.72 & -1.57 \\
\hline & $\Xi K$ & +0.50 & +1.07 & -0.57 & -0.57 \\
\hline \multirow[t]{4}{*}{$u_{\Xi^{0}}$} & $\Xi \pi$ & -0.19 & +0.19 & -0.38 & 0 \\
\hline & $\Lambda K$ & 0 & +0.25 & -0.25 & -0.25 \\
\hline & $\Sigma K$ & +4.41 & +2.21 & +2.21 & +2.21 \\
\hline & $\Omega K$ & 0 & 0 & 0 & +0.19 \\
\hline \multirow[t]{3}{*}{$d_{\Xi^{0}}$} & $\Xi \pi$ & +0.19 & +0.19 & 0 & 0 \\
\hline & $\Lambda K$ & +0.25 & +0.25 & 0 & 0 \\
\hline & $\Sigma K$ & +2.21 & +2.21 & 0 & 0 \\
\hline \multirow[t]{3}{*}{$s_{\Xi^{0}}$} & $\Lambda K$ & -0.25 & 0 & -0.25 & +0.25 \\
\hline & $\Sigma K$ & -6.62 & 0 & -6.62 & -2.21 \\
\hline & $\Omega K$ & 0 & +0.19 & -0.19 & -0.19 \\
\hline
\end{tabular}

